\title{
Partial conservation of the axial current and axial exchange currents in the nucleon
}

\author{
D. Barquilla-Cano ${ }^{1}$, A. J. Buchmann ${ }^{2}$ and E. Hernández ${ }^{1}$ \\ ${ }^{1}$ Grupo de Fisica Nuclear, Facultad de Ciencias, Universidad de Salamanca \\ Plaza de la Merced s/n, E-37008 Salamanca, Spain \\ ${ }^{2}$ Institut für Theoretische Physik, Universität Tübingen \\ Auf der Morgenstelle 14, D-72076 Tübingen, Germany
}

\begin{abstract}
We discuss the axial form factors of the nucleon within the context of the nonrelativistic chiral quark model. Partial conservation of the axial current (PCAC) imposed at the quark operator level enforces an axial coupling for the constituent quarks which is smaller than unity. This leads to an axial coupling constant of the nucleon $g_{A}$ in good agreement with experiment. PCAC also requires the inclusion of axial exchange currents. Their effects on the axial form factors are analyzed. We find only small exchange current contributions to $g_{A}$, which is dominated by the one-body axial current. On the other hand, axial exchange currents give sizeable contributions to the axial radius of the nucleon $r_{A}^{2}$, and to the non-pole part of the induced pseudoscalar form factor $g_{P}$. For the latter, the confinement exchange current is the dominant term.
\end{abstract}




\section{INTRODUCTION}

Several attempts have been made to reconcile the nonrelativistic quark model (NRQM) prediction [1] for the nucleon axial vector coupling constant

$$
g_{A}(0)=\frac{5}{3} g_{A q}(0)
$$

with the experimental value $\left(g_{A}(0) / g_{V}(0)\right)_{(\exp )}=1.2670(35)$ [2], where $g_{V}(0)=1$ according to the conserved vector current hypothesis. This $25 \%$ deviation between theory and experiment contrasts sharply with the successful NRQM prediction of nucleon magnetic moments $\mu_{p} / \mu_{n}=$ $-3 / 2$ [3] (exp.-1.46). However, many quark model calculations tacitly assume that the axial coupling of the constituent quark, $g_{A q}(0)=1$. In 1990, Weinberg [4 has offered an explanation for why $g_{A q}(0)=1$.

In 1979 Glashow [5] suggested that a deformation of the valence quark distribution in the nucleon could reduce the NRQM result of Eq.(1) while leaving the successful NRQM prediction for the nucleon magnetic moments intact [6]:

$$
g_{A}(0)=\frac{5}{3}\left(1-\frac{6}{5} P_{D}\right)
$$

where $P_{D}=0.21$ is the admixture probability of $D$-waves in the nucleon. This is a rather large $D$-state probability compared to the $P_{D}=0.0016$ calculated from gluon exchange induced tensor forces between quarks [7].

A different solution is provided by relativistic bag model calculations [8] in which

$$
g_{A}(0)=\frac{5}{3}\left(1-\frac{4}{3} \int_{0}^{\infty} d r f^{2}(r)\right)
$$

The lower component $f(r)$ of the Dirac spinor is responsible for a $30 \%$ reduction of the NRQM result. Furthermore, these bag-model calculations show that the axial vector coupling constant is completely determined by the quark core. Pion cloud effects turned out to be zero or negligibly small. This result is also obtained in a relativistic chiral constituent quark model [9]. 
Similarly, in the NRQM, the relativistic corrections to the usual one-body axial current considerably reduce $g_{A}(0)$ [10]. However, the corresponding $\mathcal{O}\left(1 / m_{q}^{2}\right)$ relativistic correction terms in the one-body electromagnetic current spoil the good agreement for baryon magnetic moments, as we have pointed out in Ref. [11]. Therefore, we have to look for a different solution of the problem within the framework of the NRQM. We will come back to this point.

In the case of the magnetic moments, the success of the NRQM is closely related to the cancellation of the various electromagnetic two-body currents required by the continuity equation

$$
\mathbf{q} \cdot \mathbf{J}(\mathbf{q})-\left[H, J^{0}(\mathbf{q})\right]=0
$$

for the electromagnetic current $\left(J^{0}, \mathbf{J}\right)$. Recently, it has been shown that the axial two-body current contributions to $g_{A}$ cancel each other [12], similar to the cancellation of the electromagnetic exchange current terms in the case of the magnetic moments. As a result of this almost complete cancellation, the NRQM prediction of Eq.(1) is not modified when axial exchange currents are included. However, the PCAC condition has not been checked in Ref. [12].

Before one can draw any conclusion concerning the failure of the NRQM to accurately predict $g_{A}(0)$ one should investigate the implications of the PCAC constraint for the axial operators at the quark level. PCAC can be formulated as [13]

$$
\mathbf{q} \cdot \mathbf{A}(\mathbf{q})-\left[H, A^{0}(\mathbf{q})\right]=-i \sqrt{2} f_{\pi} \frac{-q^{2}}{q^{2}-m_{\pi}^{2}} M^{\pi}(\mathbf{q}),
$$

where $H$ is the full Hamiltonian of the three-quark system, including the center of mass motion, $q$ is the four-momentum transfer while $\boldsymbol{q}$ stands for its spatial part. $A^{\mu}=\left(A^{0}, \mathbf{A}\right)$ is the axial current operator, $f_{\pi}=92.4 \mathrm{MeV}$ is the pion decay constant, $m_{\pi}$ the pion mass, and $M^{\pi}$ is the pion absorption/emission operator. Eq.(5) is the weak axial current analogue of Eq.(4) for the electromagnetic current. Here we will only be concerned with the non-pole part of the axial current for which the PCAC condition can be written as [13]

$$
\mathbf{q} \cdot \mathbf{A}^{\text {non-pole }}(\mathbf{q})-\left[H, A^{0, \text { non-pole }}(\mathbf{q})\right]=-i \sqrt{2} f_{\pi} M^{\pi}(\mathbf{q}),
$$


In the following we shall omit the non-pole affix when refering to the axial current in the understanding that we will always refer to its non-pole part. The PCAC condition states that the four-divergence of the axial nucleon current is not zero but proportional to the pion absorption/emission amplitude. Because the two-body terms in $H$ do not commute with the time component of the axial current $A^{0}$, the two-body potentials in $H$ must be accompanied by two-body axial exchange current and pion absorption operators if PCAC is to hold. Thus, two-body axial exchange currents consistent with the nonrelativistic quark model Hamiltonian have to be constructed, and their effect on the axial form factors of the nucleon has to be investigated. Adler and Dothan [14] have shown that the PCAC condition is as important for the axial current as Eq.(田) is for the electromagnetic current.

Even though the NRQM is a crude approximation for Quantum Chromodynamics its phenomenological success in reproducing many low energy properties of hadrons makes the present study worthwhile. We hope that some of the findings of this work will survive at least at a qualitative level in a more fundamental approach.

The paper is organized as follows. In sect. 2 we introduce the notation for the various axial form factors appearing in the general expression for the axial current. Sect. 3, briefly reviews the chiral quark potential model. The axial quark current operators are derived from Feynman diagrams and their consistency with PCAC is investigated in sect. 4. Numerical results for the axial form factors are presented and discussed in sect. 5. Finally, a short summary of the results achieved is presented.

\section{AXIAL FORM FACTORS OF THE NUCLEON}

As in the case of the electromagnetic current one can write down the allowed Lorentz structures for an axial current operator. The most general form for the nucleon axial current can be written as 15 


$$
\mathcal{A}^{\mu a}=\bar{u}^{\prime}\left(\mathbf{p}^{\prime}\right)\left(g_{A}\left(q^{2}\right) \gamma^{\mu} \gamma_{5}+2 \frac{M_{N}}{m_{\pi}^{2}} g_{P}\left(q^{2}\right) q^{\mu} \gamma_{5}+g_{T}\left(q^{2}\right) P^{\mu} \gamma_{5}\right) \frac{\boldsymbol{\tau}^{a}}{2} u(\mathbf{p}),
$$

where $u(\mathbf{p})$ and $u^{\prime}\left(\mathbf{p}^{\prime}\right)$ are the Dirac spinors of the nucleon in the initial and final state with three-momenta $\mathbf{p}$ and $\mathbf{p}^{\prime}$, and $\boldsymbol{\tau}^{a}$ is the nucleon isospin operator. The masses of the nucleon and pion are denoted by $M_{N}$ and $m_{\pi}$. Here, the $q$ is the four-momentum transfer and $P$ the total four momentum defined as

$$
q=p^{\prime}-p, \quad P=p^{\prime}+p .
$$

The three form factors in Eq.(7) $g_{A}\left(q^{2}\right), g_{P}\left(q^{2}\right)$, and $g_{T}\left(q^{2}\right)$ are scalar functions of the fourmomentum transfer. They are called the axial form factor $g_{A}$, the induced pseudoscalar form factor $g_{P}$, and the tensor form factor $g_{T}$. All three form factors are real because of the timereversal $(T)$ invariance of the weak interaction.

We work in the Breit frame where a clear separation of form factors is achieved. The nonrelativistic reduction of Eq.(7), including the normalization factors of the Dirac spinors, lead, in the Breit frame, to the following axial operators at the baryon level

$$
\begin{aligned}
A^{0}= & \frac{\boldsymbol{\sigma} \cdot \mathbf{q}}{2 M_{N}}\left(-P^{0} g_{T}\left(q^{2}\right)\right) \frac{\boldsymbol{\tau}^{+}}{2} \\
\mathbf{A}= & \boldsymbol{\sigma}\left[g_{A}\left(q^{2}\right)\left(1-\frac{\mathbf{q}^{2}}{24 M_{N}^{2}}\right)-\left(\frac{\mathbf{q}^{2}}{3 m_{\pi}^{2}} g_{P}\left(q^{2}\right)\right)\right] \frac{\boldsymbol{\tau}^{+}}{2}, \\
& +\left[\boldsymbol{\sigma}^{[1]} \otimes \mathbf{q}^{[2]}\right][1] \sqrt{\frac{5}{3}}\left(\frac{1}{8 M_{N}^{2}} g_{A}\left(q^{2}\right)+\frac{1}{m_{\pi}^{2}} g_{P}\left(q^{2}\right)\right) \frac{\boldsymbol{\tau}^{+}}{2},
\end{aligned}
$$

where $\boldsymbol{\sigma}$ and $\boldsymbol{\tau}$ are nucleon spin and isospin operators. $\boldsymbol{\tau}^{+}$is the usual ladder operator defined as $\boldsymbol{\tau}^{+}=\boldsymbol{\tau}^{x}+i \boldsymbol{\tau}^{y}$ appropriate for the $n \rightarrow p$ transition. The nonrelativistic reduction of the axial current given in Eq.(9) is equivalent to Eq.(A.7.16) in Ref. [16] if the different normalizations of the induced pseudoscalar form factor are taken into account.

Note that $g_{T}\left(q^{2}\right)$ is zero if the strong interactions are invariant under $G$-parity transformations and for transitions within the same isospin multiplet, as is the case here. This would imply that the time component of the axial current is also zero in the Breit frame. The experimental 
evidence for $g_{T}$ is very scarce. We quote here $g_{T}(0)=(-2.94 \pm 5.88) 10^{-4} \mathrm{MeV}^{-1}$ taken from Ref. [17], which is compatible with zero.

Using PCAC the induced pseudoscalar form factor can be split into two parts 14

$$
g_{P}\left(q^{2}\right)=f_{\pi} \frac{g_{\pi N N}\left(q^{2}\right)}{M_{N}} \frac{m_{\pi}^{2}}{m_{\pi}^{2}-q^{2}}+g_{P}^{\text {non-pole }}\left(q^{2}\right) .
$$

The first term is the so-called pion pole term $g_{P}^{\pi-p o l e}$. This part of the axial current is completely determined by the pion absorption amplitude and does not concern us here. For $q^{2}=0$ the non-pole part is given by

$$
g_{P}^{\text {non-pole }}(0) \simeq-\frac{1}{6} g_{A} m_{\pi}^{2} r_{A}^{2}
$$

where $r_{A}$ is the axial radius of the nucleon. This result is sometimes refered to as Adler-DothanWolfenstein (ADW) correction [18]. Eq.(11) has also been derived using chiral Ward identities and heavy baryon chiral perturbation theory [19].

Evaluating the time component of the axial current at the quark level, we obtain $g_{T}\left(q^{2}\right)$, while the spatial part of the axial operators will give us both $g_{P}^{\text {non-pole }}\left(q^{2}\right)$ and $g_{A}\left(q^{2}\right)$.

\section{THE CHIRAL QUARK MODEL}

In this section we briefly discuss the Hamiltonian of the chiral quark model $(\chi \mathrm{QM})$. The $\chi \mathrm{QM}$ was devised to effectively describe the low-energy properties of QCD. Theoretical reasons for such an approach are given in Ref. [20]. Here, the chiral symmetry of QCD is introduced via a linear $\sigma$ model with pseudoscalar $(\pi)$ and scalar $(\sigma)$ degrees of freedom. The spontaneous breakdown of chiral symmetry at the $1 \mathrm{GeV}$ scale leads to a quasi-particle picture of the constituent quark [21], which is an extended object with finite hadronic and e.m. size, and with a mass of about $1 / 3$ the mass of the nucleon. At the same time the axial coupling of constituent quarks $g_{A q}$ gets renormalized in the transition from QCD to the effective theory and deviates from its QCD value $g_{A q}=1$ as we will argue below. 
The Hamiltonian for the internal motion of three quarks with identical mass is given by:

$$
H_{\text {int }}=\sum_{j}\left(m_{q}+\frac{\boldsymbol{p}_{j}^{2}}{2 m_{q}}\right)-\frac{\boldsymbol{P}^{2}}{6 m_{q}}+\sum_{j<k}\left(\left(V^{\text {conf }}\right)_{j, k}+\left(V^{g}\right)_{j, k}+\left(V^{\pi}\right)_{j, k}+\left(V^{\sigma}\right)_{j, k}\right),
$$

where $m_{q}$ is the constituent quark mass for which we take the value $m_{q}=313 \mathrm{MeV}$. The momentum operator of the $\mathbf{j}$-th quark is denoted by $\boldsymbol{p}_{j}$, and $\boldsymbol{P}$ is the center of mass momentum of the three quark system. The kinetic energy associated with the center of mass motion is subtracted from the total Hamiltonian. Apart from the confinement potential ( $V^{\text {conf }}$ ), the Hamiltonian includes two-body interactions from one-gluon $\left(V^{g}\right)$, one-pion $\left(V^{\pi}\right)$, and one-sigma $\left(V^{\sigma}\right)$ exchange. These are obtained from the Feynman diagrams in Fig. 1. The expressions used in the following are:

$$
\begin{aligned}
& \left(V^{g}\right)_{j, k}=\frac{\alpha_{s}}{4} \boldsymbol{\lambda}_{j}^{c} \cdot \boldsymbol{\lambda}_{k}^{c}\left\{\frac{1}{r}-\frac{\pi}{m_{q}^{2}}\left(1+\frac{2}{3} \boldsymbol{\sigma}_{j} \cdot \boldsymbol{\sigma}_{k}\right) \delta(\mathbf{r})-\frac{1}{4 m_{q}^{2}}\left(3 \boldsymbol{\sigma}_{j} \cdot \hat{\mathbf{r}} \boldsymbol{\sigma}_{k} \cdot \hat{\mathbf{r}}-\boldsymbol{\sigma}_{j} \cdot \boldsymbol{\sigma}_{k}\right) \frac{1}{r^{3}}\right\} \\
& \left(V^{\pi}\right)_{j, k}=\frac{g_{\pi q}^{2}}{4 \pi} \frac{\boldsymbol{\tau}_{j} \cdot \boldsymbol{\tau}_{k}}{4 m_{q}^{2}} \boldsymbol{\sigma}_{j} \cdot \nabla_{r} \boldsymbol{\sigma}_{k} \cdot \nabla_{r}\left(\frac{e^{-m_{\pi} r}}{r}-\frac{e^{-\Lambda_{\pi} r}}{r}\right) \\
& \left(V^{\sigma}\right)_{j, k}=-\frac{g_{\sigma q}^{2}}{4 \pi}\left(\frac{e^{-m_{\sigma} r}}{r}-\frac{e^{-\Lambda_{\sigma} r}}{r}\right)
\end{aligned}
$$

where $\mathbf{r}_{j}, \boldsymbol{\sigma}_{j}, \boldsymbol{\tau}_{j}$, and $\boldsymbol{\lambda}_{j}^{c}$ are the position, spin, isospin, and color operators of the $\mathrm{j}$-th quark. The relative coordinate of the two interacting quarks is given by $\mathbf{r}=\mathbf{r}_{j}-\mathbf{r}_{k}$ with modulus $r=|\mathbf{r}|$ and unit vector $\hat{\mathbf{r}}=\mathbf{r} / r$.

The one-gluon exchange potential $V^{g}$ was suggested by de Rújula et al. [22], and later used to explain certain regularities in the spectrum of excited baryon states [23,24]. In $V^{g}$, $\alpha_{s}$ is the effective quark-gluon coupling constant, which we consider as a free parameter. Following Isgur and Karl [23] spin-orbit terms in $V^{g}$ are neglected.

In the meson exchange interactions, the quark-meson couplings $\left(g_{\pi q}, g_{\sigma q}\right)$ are related via the chiral symmetry constraint 25]

$$
g_{\sigma q}=g_{\pi q}
$$

The pion-quark coupling is determined by the experimental $\pi N$ coupling strength $f_{\pi N}^{2} / 4 \pi=0.0749$ via 27 : 


$$
\frac{g_{\pi q}^{2}}{4 \pi}=\left(\frac{3}{5}\right)^{2} \frac{f_{\pi N}^{2}}{4 \pi}\left(\frac{2 m_{q}}{m_{\pi}}\right)^{2}
$$

For the pion $\left(m_{\pi}\right)$ and sigma $\left(m_{\sigma}\right)$ masses, the bosonization technique applied to the chirally symmetric Nambu-Jona-Lasinio (NJL) Lagrangian [21] gives

$$
m_{\sigma}^{2}=4 m_{q}^{2}+m_{\pi}^{2}
$$

We use $m_{\pi}=139 \mathrm{MeV}$ from which $m_{\sigma}=641 \mathrm{MeV}$ results.

In the $\pi$ and $\sigma$ meson exchange potentials we have introduced a short distance regulator by means of the static vertex form factor

$$
F_{\pi / \sigma}\left(\boldsymbol{k}^{2}\right)=\left(\frac{\Lambda_{\pi / \sigma}^{2}-m_{\pi / \sigma}^{2}}{\Lambda_{\pi / \sigma}^{2}+\boldsymbol{k}^{2}}\right)^{1 / 2}
$$

Here, $\boldsymbol{k}$ is the three-momentum of the exchanged meson and $\Lambda_{\pi / \sigma}$ is the cut-off parameter. In coordinate space this leads to a very simple form for the potential, where a second Yukawa term with a fictitious meson mass $\Lambda_{\pi / \sigma}$ appears. For the cut-off parameters $\left(\Lambda_{\pi}, \Lambda_{\sigma}\right)$ we use

$$
\Lambda_{\pi / \sigma}^{2}=\Lambda^{2}+m_{\pi / \sigma}^{2}
$$

Both the form of the vertex form factor and the values for the cut-off parameter deviate from our previous papers (see e.g. [1],26]) where we had used $\Lambda_{\pi}=\Lambda_{\sigma}=\Lambda$ and $F\left(\mathbf{k}^{2}\right)=\left(\frac{\Lambda^{2}}{\Lambda^{2}+\mathbf{k}^{2}}\right)^{1 / 2}$. The present parametrization is best suited in order to guarantee PCAC in the presence of strong interaction vertex form factors. For $\Lambda$ we use $4.2 \mathrm{fm}^{-1}$ as obtained 27 from fitting the size of the $q \bar{q}$ component of the pion.

The constituent quarks are confined by a long-range, spin-independent, scalar two-body potential. For convenience a harmonic oscillator (h.o.) potential is often used

$$
\left(V^{\text {conf }}\right)_{j, k}=-a \boldsymbol{\lambda}_{j}^{c} \cdot \lambda_{k}^{c} r^{2}
$$

However, from lattice calculations we know that a linear confinement, which at larger distances is screened by quark-antiquark pair creation is more realistic. The effect of these color screening 
potentials on the baryon spectrum has been investigated by Zhang et al. 28. Here we consider both the standard h.o. potential and a color screening potential of the form

$$
\left(V^{c o n f}\right)_{j, k}=-a \boldsymbol{\lambda}_{j}^{c} \cdot \boldsymbol{\lambda}_{k}^{c}\left(1-e^{-\mu r}\right)+C
$$

We use the h.o. confinement potential when working with unmixed (UM) wave functions. On the other hand, a pure h.o. potential without any anharmonicity cannot reproduce the baryon mass spectrum [23,24]. Therefore, we employ a color screened confinement potential for mixed $(\mathrm{CM})$ wave functions.

The radial three-quark wave functions that we use are expanded in the harmonic oscillator basis. In the unmixed wave function approximation, the three quarks remain in their lowest h.o. state $\left|S_{S}\right\rangle$. In the configuration mixing case the wave function is not a single h.o. state but a superposition of several h.o. basis up to $N=2$ excitation quanta. With configuration mixing included, the nucleon wave function is a superposition of five h.o. states:

$$
|N\rangle=a_{S_{S}}\left|S_{S}\right\rangle+a_{S_{S}^{\prime}}\left|S_{S}^{\prime}\right\rangle+a_{S_{M}}\left|S_{M}\right\rangle+a_{D_{M}}\left|D_{M}\right\rangle+a_{P_{A}}\left|P_{A}\right\rangle
$$

A complete description of the wave functions used in Eq.(23) can be found in Ref. [24]. For a discussion on how to obtain the model parameters and the admixture coefficients in Eq.(23) we refer the reader to Refs. [26,12]. Here we just give the results for the parameters and the

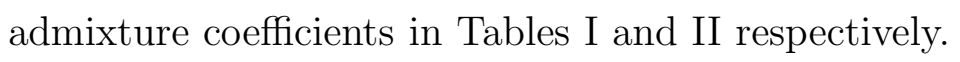

\section{THE AXIAL CURRENT OPERATORS}

\section{A. Impulse approximation}

Traditionally, in nonrelativistic quark models, the study of electromagnetic and weak properties of hadrons is done in the so-called impulse approximation, in which only one-body operators are considered. In this approximation the axial charge $\left(A^{0}\right)$ and axial current $(\mathbf{A})$ operators corresponding to Fig. 2(a) are: 


$$
\begin{aligned}
A_{i m p}^{0} & =-\frac{1}{\sqrt{2}} g_{A q} \sum_{j=1}^{3} \boldsymbol{\tau}_{j}^{1} e^{i \mathbf{q} \cdot \mathbf{r}_{j}} \frac{1}{2 m_{q}} \boldsymbol{\sigma}_{j} \cdot\left(\mathbf{q}+2 \mathbf{p}_{j}\right), \\
\mathbf{A}_{i m p} & =-\frac{1}{\sqrt{2}} g_{A q} \sum_{j=1}^{3} \boldsymbol{\tau}_{j}^{1} e^{i \mathbf{q} \cdot \mathbf{r}_{j}} \boldsymbol{\sigma}_{j}
\end{aligned}
$$

where $\mathbf{q}$ is the three-momentum transfer imparted by the $W$ boson. In the spherical basis used here, the isospin operator of the $\mathrm{j}$-th quark is given by

$$
\boldsymbol{\tau}_{j}^{ \pm 1}=\mp \frac{1}{\sqrt{2}}\left(\boldsymbol{\tau}_{j}^{x} \pm i \boldsymbol{\tau}_{j}^{y}\right)=\mp \frac{1}{\sqrt{2}} \boldsymbol{\tau}_{j}^{ \pm}, \quad \boldsymbol{\tau}_{j}^{0}=\boldsymbol{\tau}_{j}^{z},
$$

where $\boldsymbol{\tau}^{ \pm}$are usual isospin raising and lowering operators, and $\boldsymbol{\tau}_{j}^{\lambda}$ with $\lambda= \pm 1,0$ are the spherical components of the Pauli isospin matrix. We take the +1 component appropriate for the $n \rightarrow p$ transition.

As the axial current is not exactly conserved there is nothing to prevent the constituents quarks from having an effective axial charge $g_{A q}$ different from current quarks. In his second paper on $g_{A q}$ Weinberg has proven that while constituent quarks have no anomalous magnetic moments, their axial coupling may be considerably renormalized by the strong interactions [29]. In fact, explicit calculation shows that

$$
g_{A q}^{2}=1-\frac{m_{q}^{2}}{8 \pi^{2} f_{\pi}^{2}}
$$

which leads to a $8 \%$ reduction of $g_{A q}$. Weinberg's arguments have recently been re-investigated. Using Witten's large $N_{C}$ counting rules, it has been shown that, in contrast to the magnetic moment of the quarks, corrections to $g_{A q}$ appear already at order $N_{C}^{0}$ 30. Further investigation in the constituent quark structure in the NJL model shows that $g_{A q} \approx 0.78$ [31]. In a different approach, Peris 32 shows that $g_{A q}$ is renormalized by pion loops and obtains

$$
g_{A q}=1-\frac{m_{q}}{4 \pi f_{\pi}} \ln \left(\frac{m_{\sigma}^{2}}{m_{q}^{2}}\right) .
$$

Thus, by now different models of constituent quark structure agree concerning the value $g_{A q} \approx$ $3 / 4$.

As explained in the next section, a value of $g_{A q} \approx 3 / 4$ is also obtained after imposing the constraints of PCAC ( Eq.(6) ) on the axial current and pion absorption operators. 


\section{B. PCAC in impulse approximation}

In order to check PCAC for the one-body axial operators we need to know the impulse approximation contribution to the pion absorption operator. This can be derived from the Feynman diagram in Fig. 2(b) and is given by

$$
M_{i m p}^{\pi}=-i \sum_{j} \boldsymbol{\tau}_{j}^{1} \frac{g_{\pi q}}{2 m_{q}} e^{i \mathbf{q} \cdot \mathbf{r}_{j}} \boldsymbol{\sigma}_{j} \cdot \mathbf{q}
$$

It is now straightforward to prove that the relation

$$
\mathbf{q} \cdot \mathbf{A}_{i m p}-\left[T, A_{i m p}^{0}\right]=-i \sqrt{2} f_{\pi} M_{i m p}^{\pi},
$$

is satisfied 6 , up to $\mathcal{O}\left(g_{A q} / m_{q}^{2}\right)$ provided

$$
g_{A q}=f_{\pi} \frac{g_{\pi q}}{m_{q}}
$$

Thus, one obtains a Goldberger-Treiman relation at the quark level as a consequence of imposing PCAC on the axial current and pion absorption operators. Using the phenomenological pion-quark coupling from Eq.(17) $g_{\pi q}=2.62$, the empirical pion decay constant $f_{\pi}=92.4$ $\mathrm{MeV}$, and the constituent quark mass $m_{q}=313 \mathrm{MeV}$ one gets $g_{A q}=0.774$ in accord with the result [31] quoted above. If we use this value of $g_{A q}$ in the axial one-body current operators of Eq.(24) we obtain for unmixed wave functions

$$
g_{A}(0)=g_{A q} \frac{5}{3}=1.29
$$

in much better agreement with the experimental number. Obviously, the often quoted "failure of the NRQM" in reproducing $\left(g_{A}\right)_{\text {exp }}=1.267$ is related to the incorrect assumption that the axial coupling constant of the constituent quarks is the same as for current quarks, namely $g_{A q}=1$. Relativistic corrections to the one-body axial current operator are apparently not required to reproduce the empirical value for $g_{A}(0)$ in the NRQM.

\footnotetext{
${ }^{*}$ Here, $T$ is the total kinetic energy operator given by the first three terms in Eq. (12).
} 
In the following we briefly comment on our neglect of relativistic corrections of order $\mathcal{O}\left(1 / m_{q}^{2}\right)$ and higher in the one-body axial current and pion absorption operators. In our approach we include for each Feynman diagram and for each nonrelativistic invariant only the lowest nonvanishing order. As mentioned in the introduction, the inclusion of relativistic corrections in the one-body electromagnetic current spoils the successful NRQM prediction for the magnetic moments of the nucleon. See for example Eq.(6.3) and the following discussion in Ref. [11]. There, we show that including next-to-leading order relativistic corrections in the electromagnetic one-body current results in $\mu_{p}=0.89 \mu_{N}$ and $\mu_{n}=-0.59 \mu_{N}$ so that the largest part of $\mu_{p}$ would have to come from exchange currents. We argue that this is against the spirit of the nonrelativistic quark model, where the main contribution to the magnetic moments is expected to come from the single quark current. It seems that the bulk of relativistic corrections for free quark currents is already included in the leading order one-body current due to the choice of the constituent quark parameters, e.g., $m_{q}, r_{\gamma q}$, and $g_{A q}$. Therefore, we neglect next-to-leading relativistic corrections in all one-body operators. For further discussion of this

point and why the same argument does not apply to two-body operators of order $\mathcal{O}\left(1 / m_{q}^{2}\right)$ and higher see sect. III.C of Ref. [33].

The good agreement of the result in Eq.(31) seems to leave little room for configuration mixing effects and for exchange current contributions to $g_{A}(0)$. This notwithstanding, consistency requires that both effects be included in the present theory. From our analysis of axial exchange currents we will again see that the internal consistency of the constituent quark model requires $g_{A q} \approx 3 / 4$.

\section{Axial exchange current operators}

The PCAC condition not only requires a value of $g_{A q}$ different from unity, but also, as indicated in the introduction and the preceding section, the inclusion of two-body axial current and absorption operators consistent with the two-body potentials in the Hamiltonian. 
We obtain the two-body axial current and absorption operators from a nonrelativistic reduction of the Feynman diagrams of Fig. 3 and Fig. 1 respectively. We have gluon, pion and scalars ( $\sigma$ and confinement) exchange currents, plus the pion-sigma axial exchange current. As in the quark-quark potentials and the electromagnetic currents, we keep only the local terms in the operators. We hope that the nonlocal terms are to some extent included in the effective parameters of the model.

The axial gluon exchange current and absorption operators are obtained as

$$
\begin{aligned}
A_{g}^{0}= & g_{A q} \sum_{j<k} \frac{\alpha_{s}}{8 m_{q}^{2}} \boldsymbol{\lambda}_{j}^{c} \cdot \boldsymbol{\lambda}_{k}^{c}\left\{\frac{\boldsymbol{\tau}_{j}^{1}}{\sqrt{2}} e^{i \mathbf{q} \cdot \mathbf{r}_{j}}\left(\boldsymbol{\sigma}_{j} \times \boldsymbol{\sigma}_{k}\right) \cdot \mathbf{r}+(j \leftrightarrow k)\right\} \frac{1}{r^{3}} \\
\mathbf{A}_{g}= & g_{A q} \sum_{j<k} \frac{\alpha_{s}}{16 m_{q}^{3}} \boldsymbol{\lambda}_{j}^{c} \cdot \boldsymbol{\lambda}_{k}^{c}\left\{\frac { - \boldsymbol { \tau } _ { j } ^ { 1 } } { \sqrt { 2 } } e ^ { i \mathbf { q } \cdot \mathbf { r } _ { j } } \left[\left(-i\left(\boldsymbol{\sigma}_{j} \cdot \mathbf{r}\right) \mathbf{q}\right.\right.\right. \\
& \left.\left.\left.+\left(3\left(\boldsymbol{\sigma}_{j}+\boldsymbol{\sigma}_{k}\right) \cdot \hat{\mathbf{r}} \hat{\mathbf{r}}-\left(\boldsymbol{\sigma}_{j}+\boldsymbol{\sigma}_{k}\right)\right)\right) \frac{1}{r^{3}}+\frac{8 \pi}{3}\left(\boldsymbol{\sigma}_{j}+\boldsymbol{\sigma}_{k}\right) \delta(\mathbf{r})\right]+(j \leftrightarrow k)\right\} \\
M_{g}^{\pi}= & -g_{\pi q} \frac{\alpha_{s}}{8 m_{q}^{2}} \sum_{j<k} \lambda_{j}^{c} \cdot \boldsymbol{\lambda}_{k}^{c}\left\{\left(-\boldsymbol{\tau}_{j}^{1}\right) e^{i \mathbf{q} \cdot \mathbf{r}_{j}} \frac{1}{r^{3}} \boldsymbol{\sigma}_{j} \cdot \mathbf{r}+(j \leftrightarrow k)\right\}
\end{aligned}
$$

The axial pion-pair exchange current and absorption operators resulting from pseudoscalar pion-quark coupling are given next

$$
\begin{gathered}
A_{\pi}^{0}=g_{A q} \frac{g_{\pi q}^{2}}{4 \pi} \frac{1}{2 m_{q}^{2}} \sum_{j<k}\left\{\frac{\left(\boldsymbol{\tau}_{j} \times \boldsymbol{\tau}_{k}\right)^{1}}{\sqrt{2}} e^{i \mathbf{q} \cdot \mathbf{r}_{j}} \frac{1}{r} \mathcal{Y}_{1}(r) \boldsymbol{\sigma}_{k} \cdot \mathbf{r}+(j \leftrightarrow k)\right\} \\
\mathbf{A}_{\pi}=g_{A q} \frac{g_{\pi q}^{2}}{4 \pi} \frac{1}{8 m_{q}^{3}} i \sqrt{2} \sum_{j<k}\left\{e ^ { i \mathbf { q } \cdot \mathbf { r } _ { j } } \left[\boldsymbol{\tau}_{k}^{1} \frac{1}{r} \mathcal{Y}_{1}(r) \boldsymbol{\sigma}_{k} \cdot \mathbf{r} \mathbf{q}\right.\right. \\
-i\left(\boldsymbol{\tau}_{j} \times \boldsymbol{\tau}_{k}\right)^{1}\left(\boldsymbol{\sigma}_{j} \times \boldsymbol{\sigma}_{k}\right) \frac{1}{r} \mathcal{Y}_{1}(r) \\
\left.\left.+i\left(\boldsymbol{\tau}_{j} \times \boldsymbol{\tau}_{k}\right)^{1} \boldsymbol{\sigma}_{k} \cdot \mathbf{r}\left(\boldsymbol{\sigma}_{j} \times \mathbf{r}\right) \frac{1}{r^{2}} \mathcal{Y}_{2}(r)\right]+(j \leftrightarrow k)\right\} \\
M_{\pi}^{\pi}=-\frac{g_{\pi q}^{3}}{4 \pi} \frac{1}{2 m_{q}^{2}} \sum_{j<k}\left\{e^{i \mathbf{q} \cdot \mathbf{r}_{j}}\left(-\boldsymbol{\tau}_{k}^{1}\right) \frac{1}{r} \mathcal{Y}_{1}(r) \boldsymbol{\sigma}_{k} \cdot \mathbf{r}+(j \leftrightarrow k)\right\}
\end{gathered}
$$

In Eq.(33) we have used the following abbreviations

$$
\begin{aligned}
& \mathcal{Y}_{1}(r)=m_{\pi}^{2} Y_{1}\left(m_{\pi} r\right)-\Lambda_{\pi}^{2} Y_{1}\left(\Lambda_{\pi} r\right), \\
& \mathcal{Y}_{2}(r)=m_{\pi}^{3} Y_{2}\left(m_{\pi} r\right)-\Lambda_{\pi}^{3} Y_{2}\left(\Lambda_{\pi} r\right),
\end{aligned}
$$


and

$$
\begin{aligned}
& Y_{1}(x)=\frac{e^{-x}}{x}\left(1+\frac{1}{x}\right), \\
& Y_{2}(x)=\frac{e^{-x}}{x}\left(1+\frac{3}{x}+\frac{3}{x^{2}}\right) .
\end{aligned}
$$

As in the one-pion exchange potential of Eq.(14), we have introduced the short distance regulator via the static vertex form factor in Eq.(19).

The axial current and absorption operators connected with scalar exchange read

$$
\begin{aligned}
& A_{S}^{0}=0 \\
& \mathbf{A}_{S}=-\frac{1}{\sqrt{2}} \frac{i g_{A q}}{4 m_{q}^{3}} \sum_{j<k}\left\{\boldsymbol{\tau}_{j}^{1} e^{i \mathbf{q} \cdot \mathbf{r}_{j}}\left[-i\left(\boldsymbol{\sigma}_{j} \cdot \mathbf{q}\right) \mathbf{q}+\left(\mathbf{q} \cdot \boldsymbol{\nabla}_{r}\right) \boldsymbol{\sigma}_{j}-\left(\boldsymbol{\sigma}_{j} \cdot \nabla_{r}\right) \mathbf{q}\right]+(j \leftrightarrow k)\right\} V_{S}(r) \\
& M_{S}^{\pi}=i g_{\pi q} \frac{1}{2 m_{q}^{2}} \sum_{j<k}\left\{\boldsymbol{\tau}_{j}^{1} e^{i \mathbf{q} \cdot \mathbf{r}_{j}}\left(\boldsymbol{\sigma}_{j} \cdot \mathbf{q}\right)+(j \leftrightarrow k)\right\} V_{S}(r)
\end{aligned}
$$

Here, $V_{S}(r)$ stands for either the one-sigma exchange potential or the confinement potential introduced in the previous section. $A_{S}^{0}=0$ because it is purely nonlocal in lowest order. In the case of the confinement potential, which we treat as a genuine chiral scalar, there is another contribution to the pion absorption operator as explained in Ref. [34]

$$
\tilde{M}_{\text {conf }}^{\pi}=-g_{A q} \frac{1}{2 m_{q} f_{\pi}} \sum_{j<k}\left\{\boldsymbol{\tau}_{j}^{1} e^{i \mathbf{q} \cdot \mathbf{r}_{j}}\left(i \boldsymbol{\sigma}_{j} \cdot \mathbf{q}+\boldsymbol{\sigma}_{j} \cdot \nabla_{r}\right)+(j \leftrightarrow k)\right\} V_{c o n f}(r) .
$$

In order that the confinement current satisfies PCAC the latter pion absorption term has been multiplied by $g_{A q}$.

Finally, for the pion-sigma exchange diagram we get the following axial charge, current and pion absorption operators

$$
\begin{aligned}
& A_{\pi-\sigma}^{0}=0, \\
& \mathbf{A}_{\pi-\sigma}=\sqrt{2} \frac{g_{\pi q}^{2}}{4 \pi} \frac{g_{A q}}{2 m_{q}} \sum_{j<k}\left\{\boldsymbol { \tau } _ { k } ^ { 1 } \boldsymbol { \sigma } _ { k } \cdot \nabla _ { k } \left(\int_{-1 / 2}^{1 / 2} d v e^{i \mathbf{q} \cdot(\mathbf{R}-\mathbf{r} v)}\right.\right. \\
& \left.\left.\left[\left(\mathbf{r}+i \mathbf{q} r v \frac{1}{L_{v}}\right) \frac{e^{-L_{v} r}}{r}-\left(\mathbf{r}+i \mathbf{q} r v \frac{1}{\tilde{L}_{v}}\right) \frac{e^{-\tilde{L}_{v} r}}{r}\right]\right)+(j \leftrightarrow k)\right\} \\
& M_{\pi-\sigma}^{\pi}=-\frac{g_{\pi q}^{3}}{4 \pi} \frac{m_{\sigma}^{2}-m_{\pi}^{2}}{4 m_{q}^{2}} \sum_{j<k}\left\{\boldsymbol{\tau}_{k}^{1} \boldsymbol{\sigma}_{k} \cdot \boldsymbol{\nabla}_{k}\left(\int_{-1 / 2}^{1 / 2} d v e^{i \mathbf{q} \cdot(\mathbf{R}-\mathbf{r} v)}\left[\frac{e^{-L_{v} r}}{L_{v}}-\frac{e^{-\tilde{L}_{v} r}}{\tilde{L}_{v}}\right]\right)+(j \leftrightarrow k)\right\},
\end{aligned}
$$


where

$$
\begin{aligned}
& \mathbf{R}=\frac{\mathbf{r}_{j}+\mathbf{r}_{k}}{2}, \\
& L_{v}^{2}=m_{\sigma}^{2}\left(v+\frac{1}{2}\right)+m_{\pi}^{2}\left(\frac{1}{2}-v\right)+\mathbf{q}^{2}\left(\frac{1}{4}-v^{2}\right), \quad \tilde{L}_{v}^{2}=\Lambda_{\sigma}^{2}\left(v+\frac{1}{2}\right)+\Lambda_{\pi}^{2}\left(\frac{1}{2}-v\right)+\mathbf{q}^{2}\left(\frac{1}{4}-v^{2}\right) .
\end{aligned}
$$

Here we have followed the prescription for modifying the currents in the presence of strong interaction form factors as given in Ref. [35]. Note that $\mathbf{A}_{\pi-\sigma}$ contains a factor $g_{A q}$, which is required in order to satisfy $\mathrm{PCAC}$ as we will see in the next section.

\section{PCAC and exchange currents}

The axial exchange currents and pion absorption operators have been listed in the preceding section but their consistency with PCAC remains to be considered. Here, we check to what extent the axial current and pion absorption operators derived from the Feynamn diagrams in Figs.2- 1 satisfy the PCAC condition of Eq.(6).

We start with the one-gluon exchange interaction. Considering $g_{A q}$ as a $\mathcal{O}\left(1 / m_{q}\right)$ quantity, as suggested by Eq.(30), one immediately gets

$$
\begin{aligned}
{\left[T, A_{g}^{0}\right] } & =\mathcal{O}\left(1 / m_{q}^{4}\right), \\
\mathbf{q} \cdot \mathbf{A}_{g} & =\mathcal{O}\left(1 / m_{q}^{4}\right),
\end{aligned}
$$

while

$$
M_{g}^{\pi}=\mathcal{O}\left(1 / m_{q}^{2}\right)
$$

Ignoring the $\mathcal{O}\left(1 / m_{q}^{4}\right)$ contributions in Eq.(39), PCAC requires that to order $\mathcal{O}\left(1 / m_{q}^{2}\right)$

$$
\sqrt{2} i f_{\pi} M_{g}^{\pi}=\left[\sum_{j<k}\left(V_{g}\right)_{j k}, A_{i m p}^{0}\right]
$$

is satisfied. The commutator is given as

$$
\left[\sum_{j<k}\left(V_{g}\right)_{j k}, A_{i m p}^{0}\right]=-i \sum_{j<k} g_{A q} \frac{\alpha_{s}}{4 m_{q}} \boldsymbol{\lambda}_{j}^{c} \cdot \boldsymbol{\lambda}_{k}^{c}\left\{\frac{-\boldsymbol{\tau}_{j}^{1}}{\sqrt{2}} e^{i \mathbf{q} \cdot \mathbf{r}_{j}} \frac{1}{r^{3}} \boldsymbol{\sigma}_{j} \cdot \mathbf{r}+(j \leftrightarrow k)\right\},
$$


where terms of higher order than $\mathcal{O}\left(1 / m_{q}^{2}\right)$ are neglected. Comparing Eq.(41) with the pion absorbtion operator in Eq.(32) we see that Eq.(40) is satisfied in this order if $g_{A q}=f_{\pi} \frac{g_{\pi q}}{m_{q}}$ holds. Thus, the PCAC constraint for the axial gluon exchange current leads again to the GoldbergerTreiman relation as in Eq.(30). Note that the commutator in Eq.(40), and those appearing in the following, also generate higher order three-body operators. These are not taken into account here. Several recent investigations have shown that the three-body current contributions to nucleon electromagnetic properties amount to at most $30 \%$ of the two-body current contribution [36]. The issue of three-body potentials and currents needs further investigation [37.

We turn now to the pion and sigma exchange interactions. A direct calculation shows that

$$
\begin{aligned}
{\left[T, A_{\pi}^{0}\right] } & =\mathcal{O}\left(1 / m_{q}^{4}\right), \\
\mathbf{q} \cdot \mathbf{A}_{\pi} & =\mathcal{O}\left(1 / m_{q}^{4}\right), \\
M_{\pi}^{\pi} & =\mathcal{O}\left(1 / m_{q}^{2}\right),
\end{aligned}
$$

while

$$
\left[\sum_{j<k}\left(V_{\pi}\right)_{j k}, A_{i m p}^{0}\right]=\mathcal{O}\left(1 / m_{q}^{4}\right)
$$

This clearly shows that pion exchange terms alone are not sufficient to satisfy the PCAC condition. Neither the commutator nor the spatial divergence generates a $\mathcal{O}\left(1 / m_{q}^{2}\right)$ term that would correspond to the pion absorption operator in Eq.(33).

Similarly, one finds for the one-sigma exchange terms (we replace $S \rightarrow \sigma$ in Eq.(36))

$$
\begin{aligned}
{\left[T, A_{\sigma}^{0}\right] } & =\mathcal{O}\left(1 / m_{q}^{4}\right), \\
\mathbf{q} \cdot \mathbf{A}_{\sigma} & =\mathcal{O}\left(1 / m_{q}^{4}\right) .
\end{aligned}
$$

In addition, to order $\mathcal{O}\left(1 / m_{q}^{2}\right)$

$$
\left[\sum_{j<k}\left(V_{\sigma}\right)_{j k}, A_{i m p}^{0}\right]=-i \frac{1}{\sqrt{2}} \frac{g_{A q}}{m_{q}} \sum_{j<k}\left\{\boldsymbol{\tau}_{k}^{1} e^{i \mathbf{q} \cdot \mathbf{r}_{k}} \boldsymbol{\sigma}_{k} \cdot \nabla_{k}+(j \leftrightarrow k)\right\} V_{\sigma}(r),
$$

whereas 


$$
\sqrt{2} i f_{\pi} M_{\sigma}^{\pi}=-\frac{1}{\sqrt{2}} f_{\pi} \frac{g_{\pi q}}{m_{q}^{2}} \sum_{j<k}\left\{\boldsymbol{\tau}_{k}^{1} e^{i \mathbf{q} \cdot \mathbf{r}_{k}} \boldsymbol{\sigma}_{k} \cdot \mathbf{q}+(j \leftrightarrow k)\right\} V_{\sigma}(r) .
$$

Thus, as in the pion exchange case, the sigma exchange terms alone are not sufficient to recover PCAC. However, everything falls into place once the pion-sigma exchange contribution in Eq. (38) is considered. In that case

$$
\mathbf{q} \cdot \mathbf{A}_{\pi-\sigma}-\left[\sum_{j<k}\left(V_{\sigma}\right)_{j k}, A_{i m p}^{0}\right]=-i \sqrt{2} f_{\pi}\left\{M_{\pi-\sigma}^{\pi}+M_{\pi}^{\pi}+M_{\sigma}^{\pi}\right\}
$$

is satisfied to order $\mathcal{O}\left(1 / m_{q}^{2}\right)$ whenever the quark level Goldberger-Treiman relation Eq.(30) holds.

Turning to the confinement interaction one finds

$$
\begin{aligned}
{\left[T, A_{\text {conf }}^{0}\right] } & =\mathcal{O}\left(1 / m_{q}^{4}\right), \\
\mathbf{q} \cdot \mathbf{A}_{\text {conf }} & =\mathcal{O}\left(1 / m_{q}^{4}\right),
\end{aligned}
$$

and in addition to order $\mathcal{O}\left(1 / m_{q}^{2}\right)$ we find

$$
\left[\sum_{j<k}\left(V_{\text {conf }}\right)_{j k}, A_{i m p}^{0}\right]=\sqrt{2} i f_{\pi}\left(M_{c o n f}^{\pi}+\tilde{M}_{c o n f}^{\pi}\right),
$$

once again only if $g_{A q}=f_{\pi} \frac{g_{\pi q}}{m_{q}}$. The extra term $\tilde{M}_{\text {conf }}^{\pi}$ (see Eq.(37)) arises from treating the confinement interaction as a chiral scalar1 [34].

In summary, provided the Goldberger-Treiman relation at the quark level in Eq.(30) is taken into account, all the axial currents considered here satisfy PCAC to leading order.

\section{RESULTS AND DISCUSSIONS}

In Table [I] we give the results for $g_{A}(0), g_{P}^{\text {non-pole }}(0)$ and $g_{T}\left(q^{2}\right)$. In the configuration mixing case, we have not considered the small D- and P-wave state contributions. Starting with $g_{T}\left(q^{2}\right)$ we obtain exactly 0 for all values of $q^{2}$. This holds both with unmixed and mixed wave functions, in impulse approximation or for the total axial current including two-body exchange currents. This is a welcome result and a reflection of the $S U(2)$ isospin symmetry underlying our model. 


\section{A. The axial form factor $g_{A}\left(\mathbf{q}^{2}\right)$}

Turning now to $g_{A}(0)$, we find that its value is dominated by the one-body axial current. This is true both for mixed and unmixed wave functions. Different exchange currents contributions cancel to a large extent giving rise to a small $3-7 \%$ increase in the total value. Also the effect of the wave function is very small with variations of the order of $2 \%$ (see table III). These results are in good agreement with experiment.

The axial radius is discussed next. It is defined as the slope of the axial form factor

$$
r_{A}^{2}=-\left.\frac{6}{g_{A}(0)} \frac{d g_{A}\left(q^{2}\right)}{d q^{2}}\right|_{q^{2}=0}
$$

and has been measured in (quasi)elastic scattering of (anti)neutrinos on nucleons and from

charged pion electroproduction on protons. A one-parameter dipole form is used for $g_{A}\left(q^{2}\right)$ 38

$$
g_{A}\left(q^{2}\right)=g_{A}(0) \frac{1}{\left(1-q^{2} / M_{A}^{2}\right)^{2}}
$$

$M_{A}$ is the so called axial mass which is fitted to experiment. From Eq.(51) one obtains using the definition in Eq. $(50)$

$$
r_{A}^{2}=\frac{12}{M_{A}^{2}}
$$

The world averages for $r_{A}^{2}$ extracted from Ref. [39] are: $r_{A}^{2}=(0.444 \pm 0.015) \mathrm{fm}^{2}$ from neutrino reactions and $r_{A}^{2}=(0.449 \pm 0.031) \mathrm{fm}^{2}$ from pion production reactions. The latter number contains the chiral correction evaluated in [40]. When the conserved vector current hypothesis is relaxed, a two-parameter fit of $M_{A}$ and the vector mass $M_{V}$ gives generally larger values for the axial radius (see e.g. table 4 in Ref. [38). Our results are compiled in Table एV under $\left(r_{A}^{2}\right)_{0}$. In contrast to $g_{A}(0)$, we find that exchange currents give a sizeable contribution to the axial radius which amounts to $25 \%$ of the total in the unmixed case and $44 \%$ in the configuration mixing case.

The reader may object that we have not considered the possible $q^{2}$ dependence associated with $g_{A q}$ itself. In our previous work [11,26], we assumed a vector meson dominance form factor 
for the photon-quark coupling. Here, it would seem just as appropriate to use for $g_{A q}$ a form factor as given by axial-vector meson dominance [41]. This has already been done with good results at the nucleon level 42. One then has

$$
g_{A q}\left(q^{2}\right)=\frac{g_{A q}}{1-q^{2} / m_{a_{1}}^{2}}
$$

with $m_{a_{1}}=1260 \mathrm{MeV}$. This leads to an axial radius of a constituent quark

$$
r_{A q}^{2}=-\left.\frac{6}{g_{A q}(0)} \frac{d g_{A q}\left(q^{2}\right)}{d q^{2}}\right|_{q^{2}=0}=\frac{6}{m_{a_{1}}^{2}}
$$

and a numerical value $r_{A q}^{2}=0.147 \mathrm{fm}^{2}$.

With the axial form factor of the constituent quark included we get larger values for the axial radius $r_{A}^{2}$ as shown in table $\mathbb{I V}$. Obviously, the impulse approximation agrees better with the data. The total result, including exchange currents, gives an axial nucleon radius close to the electromagnetic radius of the proton, i.e., a value that is too large compared with present experimental data. The reason for this deviation between theory and experiment is mainly due to the confinement contribution to the axial current.

In Figs. 5 and 6 we show our results for $g_{A}\left(q^{2}\right) / g_{A}(0)$, with and without configuration mixing respectively. We have included the $q^{2}$ dependence of $g_{A q}$ as given by axial-vector meson dominance. We compare our impulse approximation (dotted line) and our total results (solid line) with experimental data and with the dipole fit (dashed line), using for the latter an axial mass given by $M_{A}=1.025 \mathrm{GeV}$. We see that in the very low $q^{2}$ region the data are best described when exchange currents are included, although the absence of data points below 0.08 $\mathrm{GeV}^{2}$ and the big error bars do not allow to be very conclusive. At higher momentum transfers, exchange currents give sizeable contributions that worsen the agreement with the data. We also show another line (dashed-dotted) that corresponds to our total results but with no $q^{2}$ dependence in $g_{A q}$. A better agreement with data is achieved in this latter case. 


\section{B. The induced pseudoscalar form factor $g_{P}\left(\mathbf{q}^{2}\right)$}

The non-pole contribution $g_{P}^{\text {non-pole }}(0)$ to the pseudoscalar coupling constant is mainly given by exchange currents, and its value is dominated by the axial confinement current. This form factor is sensitive to the wave function, being some $25 \%$ larger in absolute value with configuration mixing. The predictions in Table $\amalg$ agree in sign and magnitude with the ADW result in Eq.(11). The same holds true for another chiral quark model calculation [9] when their $G_{P}^{\text {non-pole }}(0)$ is divided by the factor $-\left(2 M_{N} / m_{\pi}\right)^{2}$ in order to obtain our normalization. There is a proposal to measure $g_{P}$ at PSI with a $2 \%$ accuracy 45. This would allow to separate the non-pole from the dominant pion pole contribution to $g_{P}$ and to test recent quark model predictions for this quantity.

\section{CONCLUSIONS}

In the present paper we have investigated the three axial form factors $g_{A}\left(q^{2}\right), g_{P}\left(q^{2}\right)$, and $g_{T}\left(q^{2}\right)$ in the nonrelativistic chiral quark model paying attention to the implications of the PCAC condition for the axial current.

The PCAC relation requires an effective axial coupling of the constituent quarks $g_{A q} \approx 0.75$. As a result, the nucleon axial coupling, $g_{A}$, evaluated in the quark model, is in good agreement with experiment without invoking $D$-states or lower components in Dirac spinors.

The PCAC relation also requires that axial exchange currents consistent with the interquark potentials be included in the theory. We have investigated the influence of exchange currents on the axial form factors of the nucleon. We find that exchange currents do not contribute significantly to $g_{A}(0)$. However, they have a sizeable effect on the axial radius. When combined with axial-vector meson dominance we get axial nucleon radii that are too large compared to the experimental data.

We have seen that axial exchange currents, in particular the confinement axial current, also 
give the main contribution to the non-pole part of the induced pseudoscalar coupling constant $g_{P}^{\text {non-pole }}(0)$. Our prediction agrees in sign and magnitude with the Adler-Dothan-Wolfenstein result.

For $g_{T}\left(q^{2}\right)$ we obtain exactly 0 for all values of $q^{2}$.

\section{ACKNOWLEDGMENTS:}

A.J.B. thanks J. Adam jr. for useful correspondence and K. Tsushima for calculational help in the inital stages of the project. We also thank M. R. Robilotta for useful correspondence concerning the treatment of chiral scalar interactions. D. Barquilla-Cano thanks the Junta de Castilla y Leon for a predoctoral fellowship. Work supported in part by Spanish DGICYT under contract no. BFM2000-1326 and Junta de Castilla y Leon under contract no. SA109/01. 
[1] R. van Royen and V. F. Weisskopf, Nuovo Cimento 50 A, 617 (1967).

[2] D.E. Groom et al., Eur. Phys. J. C 15, 1 (2000).

[3] M. A. B. Beg, B. W. Lee, and A. Pais, Phys. Rev. Lett. 13, 514 (1964).

[4] S. Weinberg, Phys. Rev. Lett. 65, 1181 (1990).

[5] S. L. Glashow, Physica 96 A, 27 (1979).

[6] There is an analogy to this suggestion in nuclear physics. In impulse approximation, the axial coupling of triton is given in terms of the free neutron axial coupling constant as follows [13]: $G_{A}(0)=-g_{A}(0)\left(P_{S}-1 / 3 P_{S^{\prime}}+1 / 3 P_{D}\right)$, where $P_{S}, P_{S^{\prime}}$, and $P_{D}$ are the symmetric $S$-wave and the mixed symmetric $S$ - and $D$-wave probabilities, respectively, which satisfy $P_{S}+P_{S^{\prime}}+P_{D}=1$. The deviation of the triton wave function from a pure $S$-state leads to some reduction of the axial coupling of ${ }^{3} H$ with respect to $g_{A}(0)$ of the free neutron.

[7] N. Isgur, G. Karl, R. Koniuk, Phys. Rev. D 25, 2394 (1982).

[8] R. Tegen, M. Schedl and W. Weise, Phys. Lett. B 125, 9 (1983); R. Tegen and W. Weise, Z. Phys. A314, 357 (1983).

[9] S.Boffi, L. Ya. Glozman, W. Klink, W. Plessas, M. Radici and R. F. Wagenbrunn, Eur. Phys. J. A14, 17 (2002).

[10] K. Dannbom, L. Ya. Glozman, C. Helminen and D. O. Riska, Nuc. Phys. A616, 555 (1997).

[11] A. Buchmann, E. Hernández, and K. Yazaki, Nucl. Phys. A569, 661 (1994), Phys. Lett. 269, 35 (1991).

[12] A. J. Buchmann, Z. Naturforsch. 52a, 877 (1997).

[13] J. Adam, Ch. Hajduk, H. Henning, P. U. Sauer, E. Truhlik, Nucl. Phys. A531,623 (1991). 
[14] S. L. Adler and Y. Dothan, Phys. Rev. 151, 1267 (1966).

[15] M. Gourdin, Phys. Rep. 11, 29 (1974).

[16] T. E. O. Ericson and W. Weise, Pions and Nuclei, Clarendon Press, Oxford, 1988.

[17] W. Weise, "Quarks and Nuclei", International Review of Nuclear Physics, Vol. 1 (1984), p. 117. edited by W. Weise.

[18] V. Bernard, L. Elouardrhiri and Ulf-G Meissner, J. Phys. G28, R1 (2002); N. C. Mukhopadhyay in: Santa Fe 1998, Physics beyond the standard model, ed. P. Herczeg et al. (World Scientific, Singapore 1999) p.222; see also nucl-th/9810039; see also L. Wolfenstein, in: High Energy Physics and Nuclear Structure, ed. S. Devons (Plenum, New York, 1970) p.661.

[19] V. Bernard, N. Kaiser, U. G. Meissner, Phys. Rev. D 50, 6899 (1994).

[20] A. Manohar and H. Georgi, Nucl. Phys. B 234, (1984)189.

[21] U. Vogl and W. Weise, Prog. Part. Nucl. Phys. 27, 195 (1991).

[22] A. de Rújula, H. Georgi and S.L. Glashow, Phys. Rev. D 12, 147 (1975).

[23] N. Isgur and G. Karl, Phys. Rev. D 18, 4187 (1978); N. Isgur, G. Karl and R. Koniuk, Phys. Rev. Lett. 41, 1269 (1978).

[24] N. Isgur and G. Karl, Phys. Rev. D 19, 2653 (1979).

[25] I. T. Obukhovsky, A. M. Kusainov, Phys. Lett. B 238, 142 (1990).

[26] U. Meyer, E. Hernández and A. J. Buchmann, Phys. Rev. C 64, 035203 (2001).

[27] F. Fernández and E. Oset, Nucl. Phys. A455, 720 (1986).

[28] Zong-Ye Zhang, You-Wen Yu, Peng-Nian Shen, Xiao-Yan Shen and Yu-Bin Dong, Nucl. Phys. A561, 595 (1993).

[29] S. Weinberg, Phys. Rev. Lett. 67, 3437 (1991).

[30] W. Broniowski, M. Lutz and A. Steiner, Phys. Rev. Lett. 71, 1787 (1993). 
[31] U. Vogl, M. Lutz, S.Klimt and W. Weise, Nucl. Phys. A516, 469 (1990).

[32] S. Peris, Phys. Lett. B 268, 415 (1991).

[33] A. J. Buchmann, E. Hernández, U. Meyer, A. Faessler, Phys. Rev. C 58, 2478 (1998).

[34] C. M. Maekawa, and M. R. Robilotta, Phys. Rev. C57, 2839 (1998).

[35] J. Adam and H. Arenhövel, Nucl. Phys. A614, 289 (1997).

[36] G. Dillon and G. Morpurgo, Phys. Lett. B 448, 107 (1999); A. J. Buchmann and R. Lebed, Phys. Rev. D 62, 096005 (2000).

[37] V. Dmitrašinović, Phys. Lett. B 499, 135 (2001).

[38] T. Kitagaki et al., Phys. Rev. D 42, 1331 (1990). Possible problems with the assumption of a dipole form in the low-momentum tranfer region are mentioned.

[39] A. Liesenfeld et al., Phys. Lett. B 468, 19 (1999).

[40] V. Bernard, N. Kaiser and Ulf G. Meissner, Phys. Rev. Lett. 69, 1877 (1992).

[41] The authors of Ref. 31] find an axial quark radius of $0.036 \mathrm{fm}^{2}$, and that the ratio of the electromagnetic and axial quark radius is approximately given by $m_{a_{1}} / m_{\rho}$, where $m_{a_{1}}$ and $m_{\rho}$ are the masses of the $a_{1}$ axial-vector and $\rho$ vector meson respectively. This ratio is also obtained from the vector meson dominance model.

[42] M. Gari and U. Kaulfuss, Phys. Lett. B 138, 29 (1984).

[43] W. Weise in "Quarks and Nuclei", International Review of Nuclear Physics, Vol. 1 (1984), p. 121. edited by W. Weise.

[44] A. Manohar and H. Georgi, Nucl. Phys. B 234, 189 (1984).

[45] D. V. Balin et al., PSI proposal R-97-05. 
TABLE I. Quark model parameters. Set I: for quadratic confinement and unmixed wave functions. Set II: for color screening confinement and configuration mixed wave functions. $\mathrm{b}$ is the harmonic oscillator constant, $\alpha_{s}$ is the quark-gluon coupling strength, a is the confinement strength, $\mu$ the color screening length, and $\mathrm{C}$ a constant term in the color screening confinement potential.

\begin{tabular}{lccccc}
\hline \hline & $b[\mathrm{fm}]$ & $\alpha_{s}$ & $a$ & $\mu\left[\mathrm{fm}^{-1}\right]$ & $C[\mathrm{MeV}]$ \\
\hline Set I & 0.613 & 1.110 & $19.23\left[\mathrm{MeV} / \mathrm{fm}^{2}\right]$ & - & - \\
\hline Set II & 0.700 & 1.064 & $394.81[\mathrm{MeV}]$ & 1.824 & -769.822 \\
\hline \hline
\end{tabular}

TABLE II. Admixture coefficients in the wave function of Eq.(23), evaluated with the Hamiltonian of Eq.(12) and parameter set II in an $N=2$ harmonic oscillator space.

\begin{tabular}{lcccc}
\hline \hline$a_{S_{S}}$ & $a_{S_{S}^{\prime}}$ & $a_{S_{M}}$ & $a_{D_{M}}$ & $a_{P_{A}}$ \\
\hline 0.9033 & -0.3909 & -0.1710 & -0.0442 & 0.0006 \\
\hline \hline
\end{tabular}


TABLE III. Axial couplings $g_{A}(0), g_{P}^{\text {non-pole }}(0)$ and $g_{T}\left(q^{2}\right)$ calculated in the Breit frame. Results evaluated with unmixed (configuration mixed) wave functions are denoted by UM (CM). The individual axial current contributions are labelled as follows: Impulse (Imp.); gluon exchange (g); pion exchange $(\pi)$; sigma exchange $(\sigma)$; pion-sigma exchange $(\pi-\sigma)$; confinement (Conf.); total result (Total).

\begin{tabular}{|c|c|c|c|c|c|c|c|}
\hline & Imp. & $\mathrm{g}$ & $\pi$ & $\sigma$ & $\pi-\sigma$ & Conf. & Total \\
\hline \multicolumn{8}{|l|}{ UM } \\
\hline$g_{A}(0)$ & 1.290 & -0.221 & 0.151 & 0 & 0.091 & 0 & 1.311 \\
\hline$g_{P}^{\text {non-pole }}(0)$ & -0.0035 & 0.0104 & -0.0043 & 0.0031 & 0.0011 & -0.0313 & -0.0245 \\
\hline$g_{T}\left(q^{2}\right)\left[\mathrm{MeV}^{-1}\right]$ & 0 & 0 & 0 & 0 & 0 & 0 & 0 \\
\hline
\end{tabular}

CM

\begin{tabular}{cccccccc}
$g_{A}(0)$ & 1.257 & -0.203 & 0.162 & 0 & 0.093 & 0 & 1.309 \\
$g_{P}^{\text {non-pole }}(0)$ & -0.0034 & 0.0101 & -0.0042 & 0.0031 & 0.0012 & -0.0402 & -0.0334 \\
$g_{T}\left(q^{2}\right)\left[\mathrm{MeV}^{-1}\right]$ & 0 & 0 & 0 & 0 & 0 & 0 & 0 \\
\hline \hline
\end{tabular}


TABLE IV. Axial radius of the nucleon obtained in the Breit frame. Notation as in Table III. Results using a constant $g_{A q}$ are denoted by $\left(r_{A}^{2}\right)_{0}$. Axial radii calculated with a $q^{2}$ dependence for $g_{A q}$ as given by axial-vector meson dominance (see Eq.(53)) are denoted by $r_{A}^{2}$. The experimental results for the axial mass $M_{A}$ [39] give according to Eq. (52) $r_{A}^{2}=(0.444 \pm 0.015) \mathrm{fm}^{2}$ (from neutrino scattering) and $r_{A}^{2}=(0.449 \pm 0.031) \mathrm{fm}^{2}$ (from electro-pionproduction).

\begin{tabular}{|c|c|c|c|c|c|c|c|}
\hline & Imp. & $\mathrm{g}$ & $\pi$ & $\sigma$ & $\pi-\sigma$ & Conf. & Total \\
\hline \multicolumn{8}{|l|}{ UM } \\
\hline$\left(r_{A}^{2}\right)_{0}\left[\mathrm{fm}^{2}\right]$ & 0.364 & -0.006 & 0.008 & 0.015 & 0.027 & 0.072 & 0.480 \\
\hline$r_{A}^{2}\left[\mathrm{fm}^{2}\right]$ & 0.509 & -0.031 & 0.025 & 0.015 & 0.039 & 0.072 & 0.630 \\
\hline \multicolumn{8}{|l|}{$\mathrm{CM}$} \\
\hline$\left(r_{A}^{2}\right)_{0}\left[\mathrm{fm}^{2}\right]$ & 0.349 & -0.003 & 0.008 & 0.016 & 0.026 & 0.192 & 0.588 \\
\hline$r_{A}^{2}\left[\mathrm{fm}^{2}\right]$ & 0.490 & -0.025 & 0.027 & 0.016 & 0.036 & 0.192 & 0.736 \\
\hline
\end{tabular}




\section{Figures}

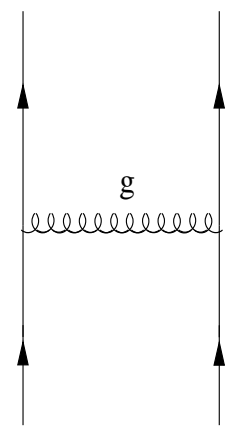

(a)

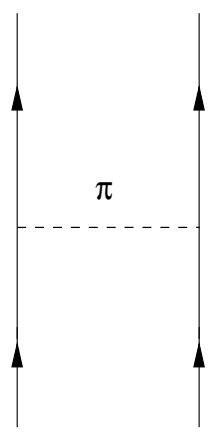

(b)

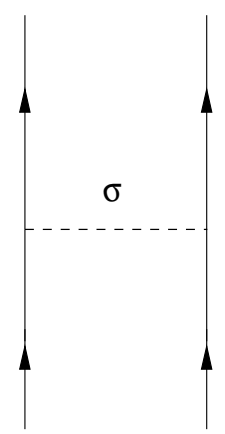

(c)

FIG. 1. Feynman diagrams for the two-body potentials: (a) one-gluon exchange potential, (b) one-pion exchange potential, (c) one-sigma exchange potential. 


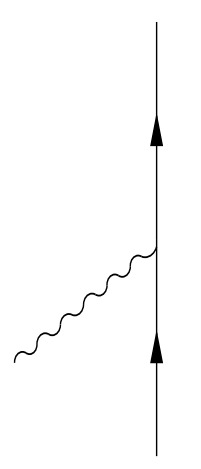

(a)

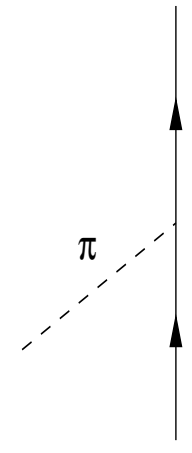

(b)

FIG. 2. Feynman diagrams for one-body operators: (a) axial current operator, (b) pion absorption operator. 


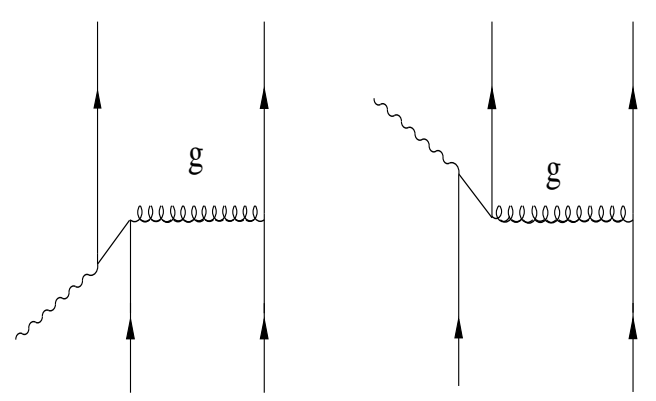

(a)

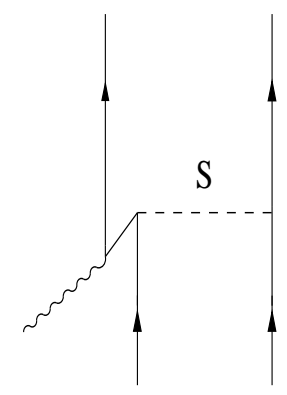

(c)

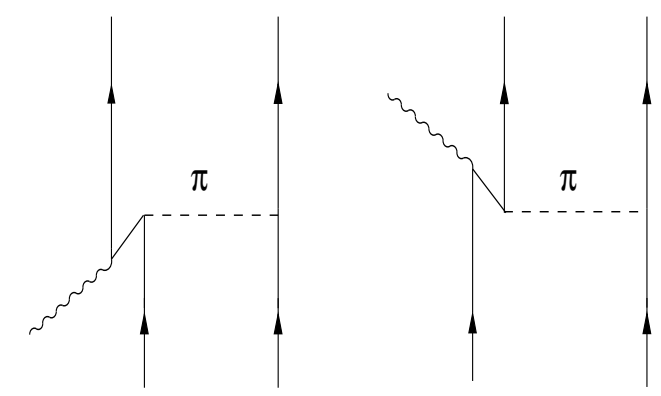

(b)

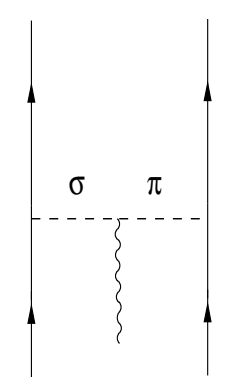

(d)

FIG. 3. Feynman diagrams for the axial exchange current operators: (a) one-gluon exchange, (b) one-pion exchange, (c) scalar exchange (sigma plus confinement), (d) pion-sigma exchange. The wavy line represents the weak gauge boson $\mathrm{W}$. 


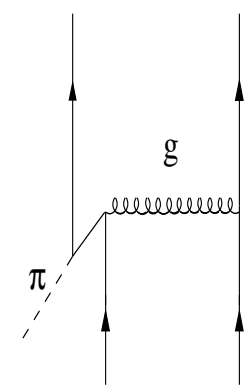

(a)

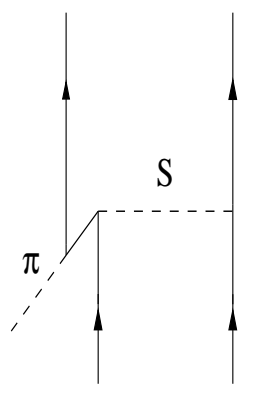

(c)

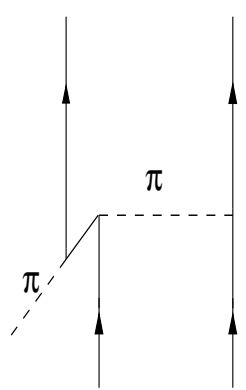

(b)

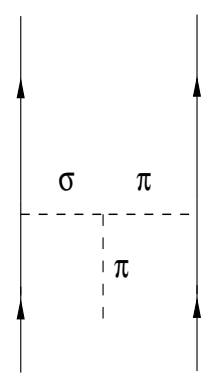

(d)
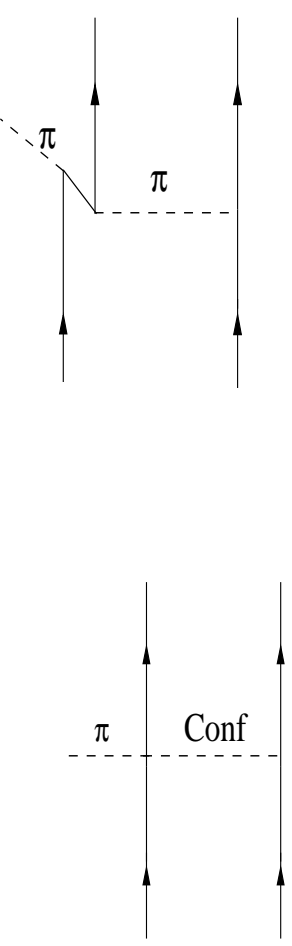

(e)

FIG. 4. Feynman diagrams of the pion absorption exchange operators: (a) one-gluon exchange, (b) one-pion exchange, (c) scalar exchange (sigma plus confinement), (d) pion-sigma exchange, (e) additional contact diagram for a chirally invariant scalar confinement interaction (see [34]). 
FIG. 5. $g_{A}\left(q^{2}\right) / g_{A}(0)$ evaluated with configuration mixing $(\mathrm{CM})$ in the nucleon wave function. With the exception of the dashed-dotted line a $q^{2}$ dependence for $g_{A q}$ as given by axial-vector meson dominance in Eq.(53) is included. The dotted line is obtained in impulse approximation. The solid line is our total result including the contribution of axial exchange currents. The dashed-dotted line is our total result calculated with a $q^{2}$-independent axial quark coupling constant $g_{A q}=0.774$. The long-dashed line is the dipole fit with $M_{A}=1.025 \mathrm{GeV}$. Experimental points are adapted from Ref. [43].

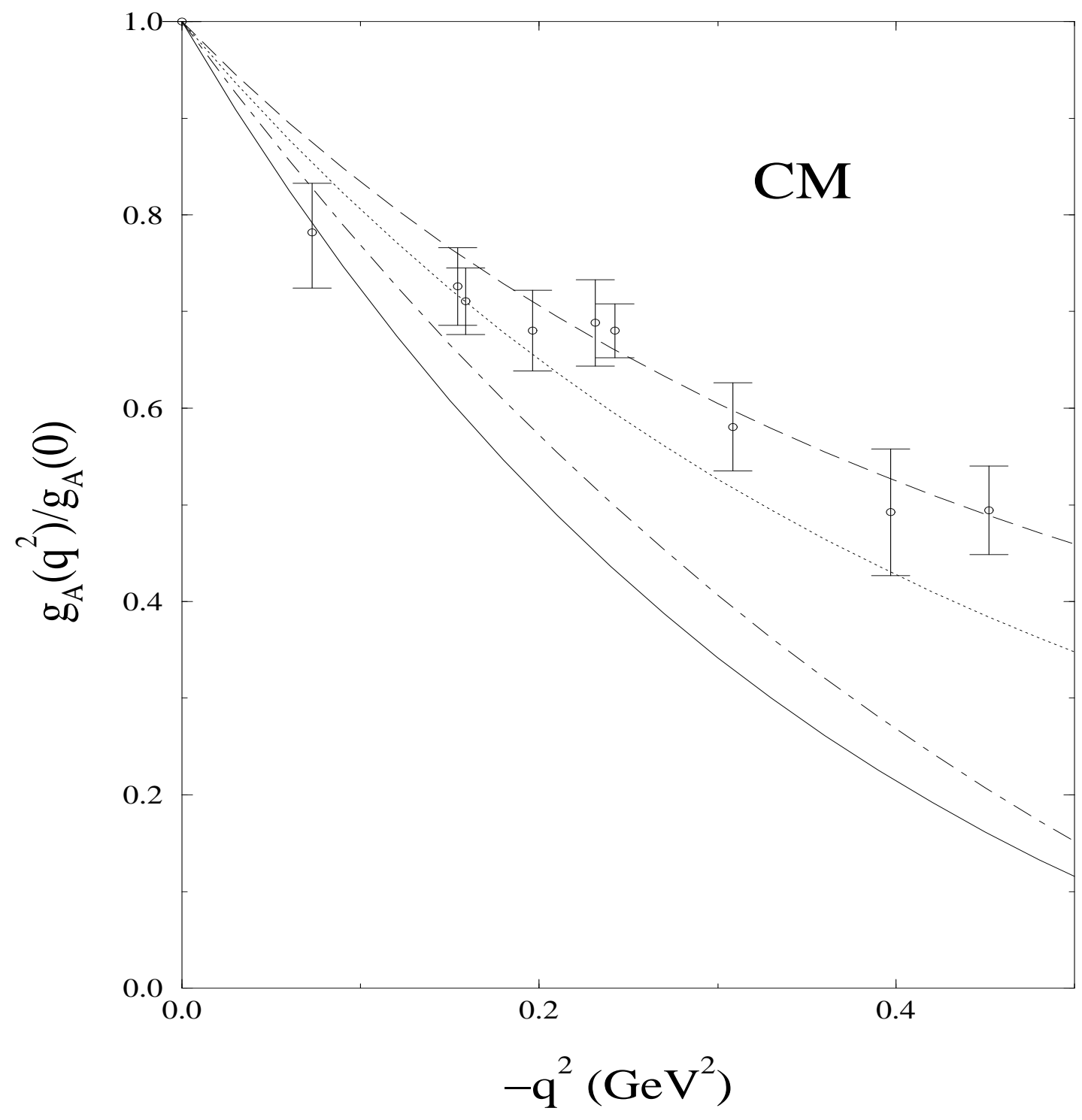


FIG. 6. $g_{A}\left(q^{2}\right) / g_{A}(0)$ evaluated without configuration mixing in the nucleon wave function (UM). Notation as in Fig. 5.

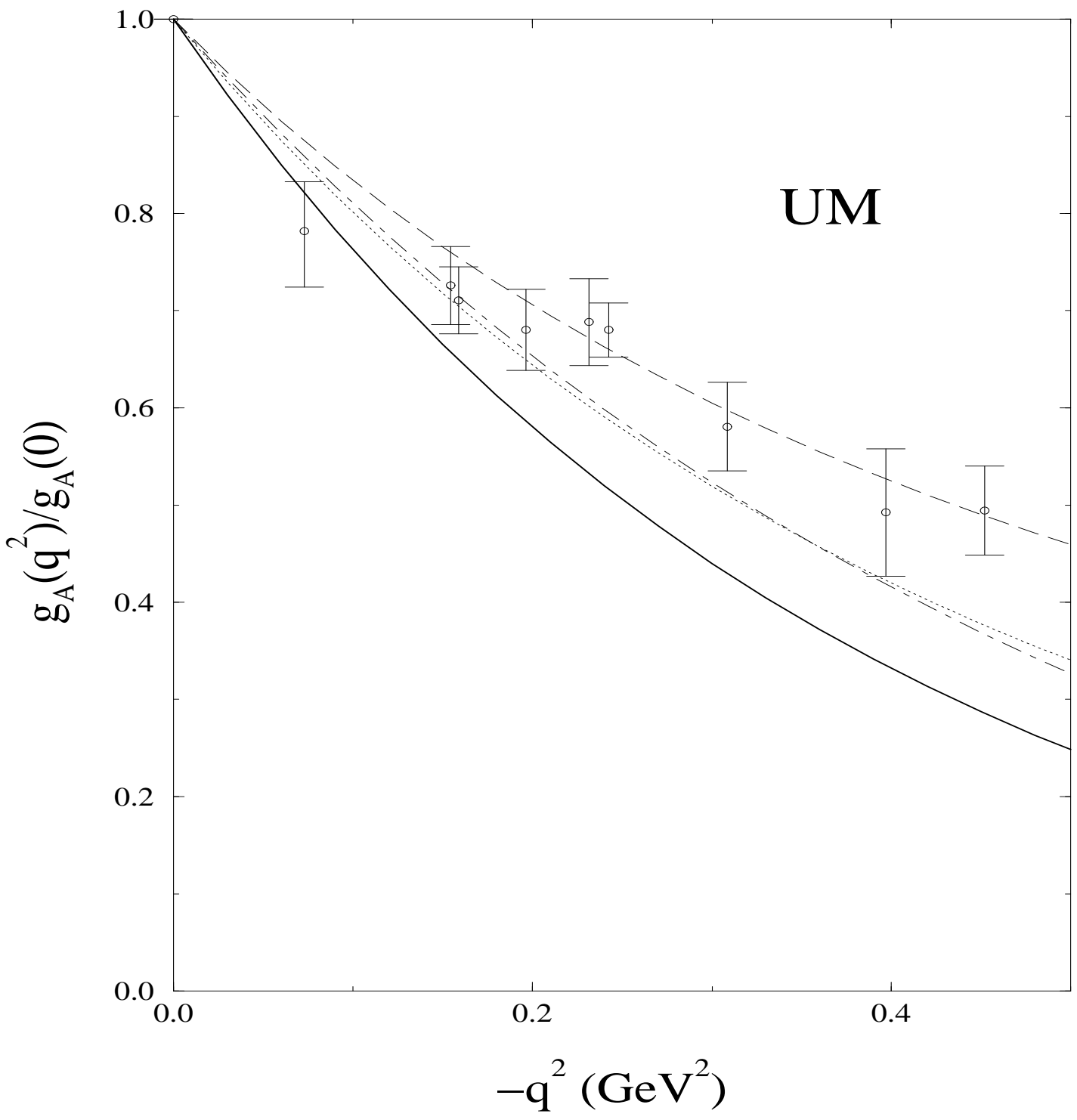

